\title{
High Speed and Intelligent Environment Monitoring System
}

\author{
Xiaohui Wang ${ }^{1,2, a^{*}}$ and Lei $\operatorname{Tian}^{3, b}$ \\ ${ }^{1}$ College of Tourism and Environment, Shaanxi Normal University, Xi'an 710119, China \\ ${ }^{2}$ Leisure Management College, Xi'an Eurasia University, Xi'an 710065, China \\ ${ }^{3}$ School of Electronic Engineering, Xi'an University of Posts and Telecommunications, \\ Xi'an 710121, China \\ awxh2324@126.con, btianlei@xupt.edu.cn
}

Keywords: MCU; Particulate matter; Carbon dioxide; Sensor; Photo detector; Remote monitor; Wireless transmission.

\begin{abstract}
In order to monitor the environmental parameter in our daily life combines the micro controller unit (MCU) and the intelligent sensor together and establishes the monitoring scheme of the particulate matter 2.5 (PM2.5) and carbon dioxide $\left(\mathrm{CO}_{2}\right)$. Based on the solar power energy, using the STC89C52 as the MCU, the PM2.5 sensor detects the concentration of the matter. The $\mathrm{CO}_{2}$ sensor detects the concentration of the carbon dioxide and through the analogue to digital convertor (ADC) converts the collected data and displays it on the LCD. At the same time, the MCU controls the wireless transmission unit to send the data to the mobile phone. Through the monitoring system, the main control platform could monitor the remote detection area. The test result shows the proposed scheme achieves better performance.
\end{abstract}

\section{Introduction}

In recent years, the hazy weather attacks most areas in our country. The serious air pollution affects our life and even threats our health. When the particulate matter inhaled into our body, it is easy to induce the serious disease [1] [2]. In order to monitor the environment in time, this paper constructs the multifunctional intelligent sensor system for collecting the actual data and transmits it by the wireless module [3] [4]. In the wild, an intelligent environment monitoring system based on the MCU is developed [5]. According to the actual requirement, the system will accomplish the remote detection with a low cost and multi-functional design scheme [6] [7]. For the unattended working environment, the cheap and sustainable power system is required. So the solar energy will be the power system [8] [9].

The system working in the unattended environment, so the detected data should send to the main control platform by the wireless network in time [10 12]. In this paper, accomplishes the wireless communication from the monitoring system to the mobile terminal by the wireless module SIM900. The system diagram is given in Fig. 1.

\section{Intelligent Sensor Monitoring Structure System}

Solar Power Block. In order to achieve the energy conservation and the environmental protection, the solar energy board is used for photovoltaic conversion. Under the extremely condition, the solar energy turns the incoming light into photocurrent and provides stable power to the whole system [13]. 


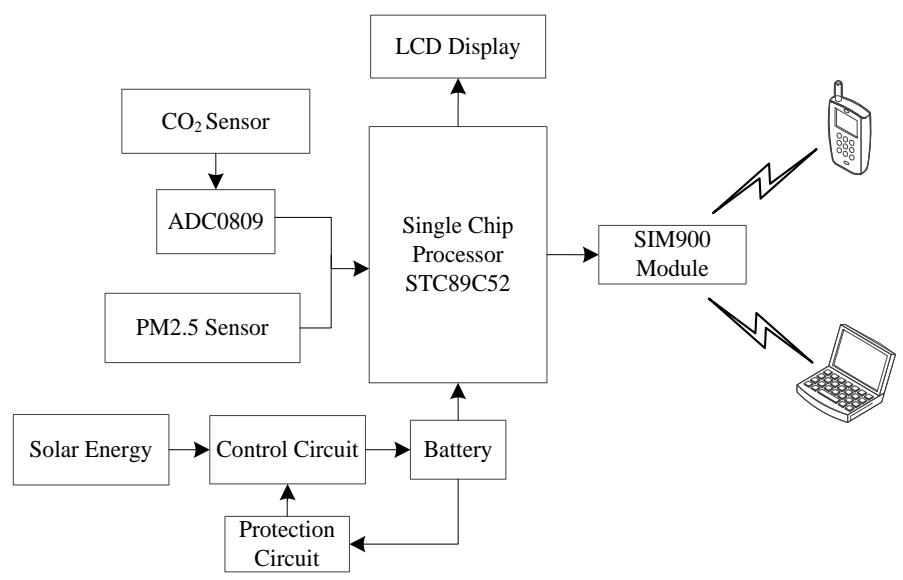

Figure 1. The Whole Structure of the System

PM2.5 Sensor Detection. According to the characteristics of the dust particulates, this paper uses the GP2Y1051AUOF sensor to detect the particulates around. In order to detect the concentration of particulates more accurately, constructed two symmetric light axes in the PM2.5 sensor. One is the LED light axis and the other is the PD light axis. These two axes diagonal build the particulate monitoring area. Dust through the circuit board in the middle of the round hole, the optical axis changes will be made by light parts to detect the space dust concentration, the sensor circuit and the light axis as shown in Fig. 2.

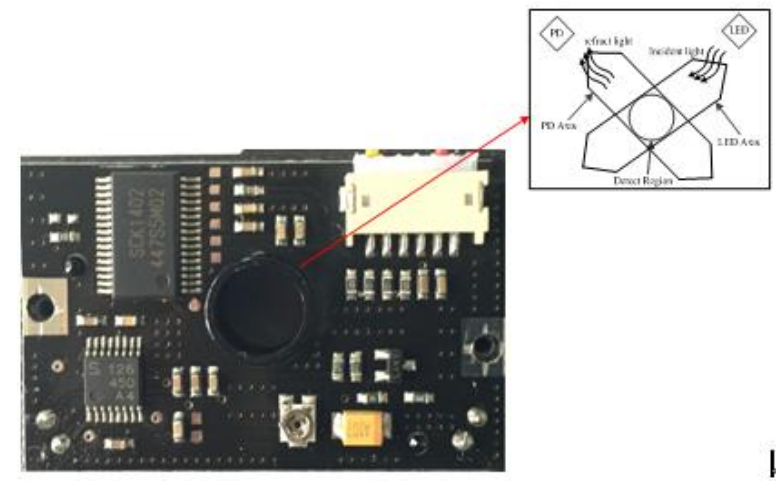

Figure 2. The PCB of the PM2.5 sensor

$\mathrm{CO}_{2}$ Sensor Detection Theory. $\mathrm{CO}_{2}$ sensor has high sensitivity within the scope of 0 10000PPM. It converts the volumetric fraction into electrical signals. It has the rapid response recovery features, the good insulation heat dissipation, low cost and high sensitivity for carbon dioxide. Through the MCU program instruction, sensor detects the $\mathrm{CO}_{2}$ concentration and sends the signal to the 26 pin to the AD chip.

Display Module. In this paper, we use the STC89C52 as the MCU and combined the LCD12864 together. The MCU transfers the collected data to the LCD and displays them in time. According to the data, we can conclude the air quality around the monitoring system. The detailed process of the block is in Fig. 3.

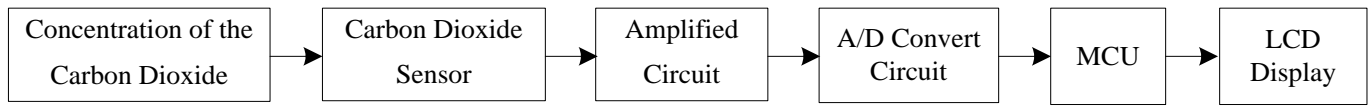

Figure 3. (a) The detecting flow chart of the $\mathrm{CO}_{2}$ concentration 


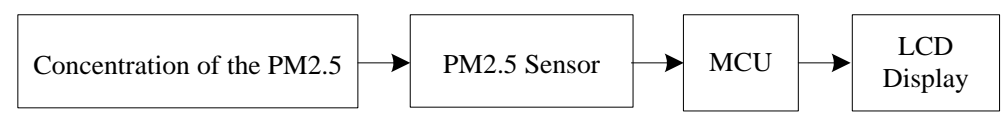

Figure 3. (b) The detecting flow chart of the PM2.5 concentration

Wireless Transmission Module.SIM900 is a wireless communication module which core is C35i. It is widely used in the communication region, such as mobile phone, short message service and GPRS. Through the P3.0 and P3.1 port, the MCU sends the transit command to the SIM900 module. When the system received the data from the PM2.5 sensor and the $\mathrm{CO}_{2}$ sensor, the SIM900 module is opened. Then the short message service and the voice communication module are used. Through the IPEX antenna interface, the SIM900 could send the collected data to the main control platform or the mobile phone.

Software Work Process. Open the whole system and initialized the LCD and the MCU. The system delays for a while and then the LCD shows the reserved words. The PM2.5 sensor collects the particulates and records the data. The MCU reads the PM2.5 numerical value and displays on the LCD.

The first step of detecting the $\mathrm{CO}_{2}$ concentration is the same with the PM2.5. The data collected by the $\mathrm{CO}_{2}$ sensor is amplified and input to the A/D conversion chip. Through the interrupt program, the system saves the data and sends it to the MCU. The MCU reads the data and displays it on the LCD. When the sensor works well and detected the environmental data, the system will start wireless module to transfer the detected data.

\section{Results and Discussion}

When the system works well, the inside SIM card in the SIM900 module opens the GPRS or GSM mode to debug the SIM900 module by serial port.

When the SIM900 module starts well, it will search the signal. At this time, the frequency of the signal lights flashing is about one time per second. In the normal condition of the mobile communications, when the SIM900 found the signal the blinking frequency will become about once every 2 seconds. The system diagram is shown in Fig. 4:

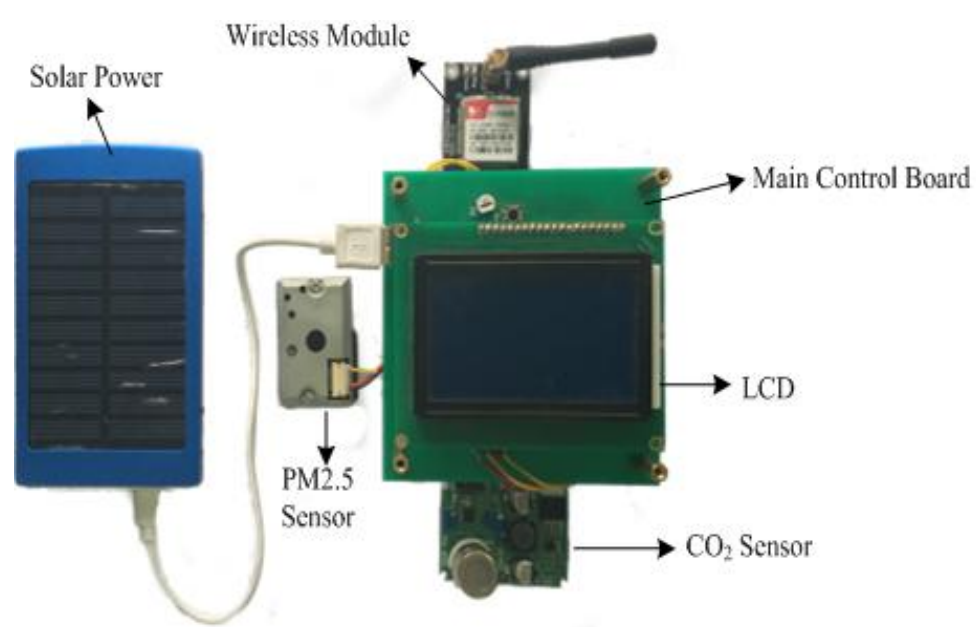

Figure 4. The connection diagram of the whole system

The MCU connects with the SIM900 and protects it works normally. Then the MCU stores the concentration of the PM2.5 and the $\mathrm{CO}_{2}$ and controls the SIM900 module sending the information to the selected mobile phone. In order to ensure the accuracy of the data transmission in the complicated 
network environment, using the TCP model and sends the command through the GPRS network. The transmitted data in the mobile phone is given by Fig. 5 .

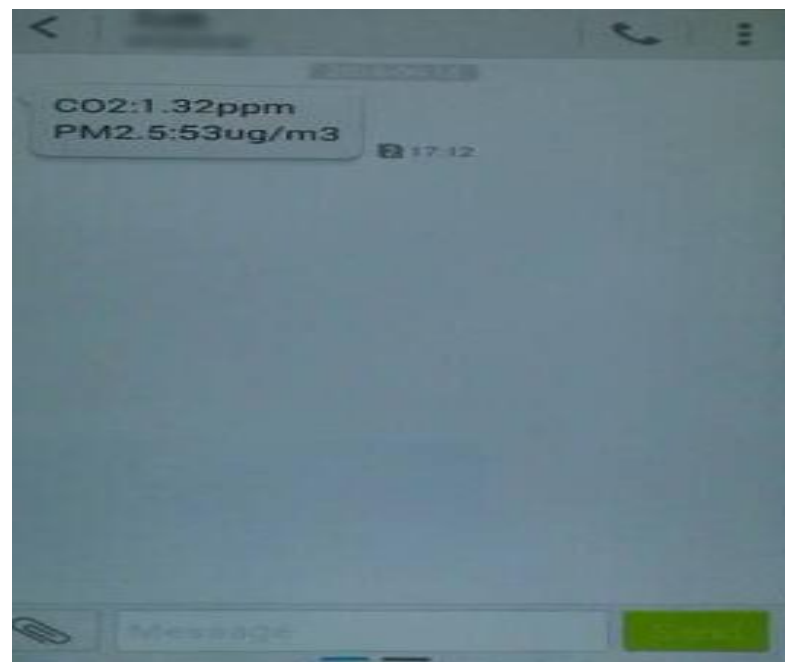

Figure 5. The test result of the monitoring system

Through the GPRS network, the different monitoring site sends the selected data to the mobile phone. The SIM900 module selects the service number to sends messages in the text mode. According different data from the monitoring sites, the main control platform could establish the database and monitoring the spots in time.

\section{Conclusions and Future Development}

Based on the solar energy, the system used the PM2.5 sensor and the carbon dioxide sensor to monitor the concentration of them. When the data is collected, the data would be sent to the mobile phone. Through the test data, it shows that this system could monitor the air quality by the intelligent sensor to guarantee the current air quality level transmitted to the user in time. In addition, this system has the characteristics of low cost, long life and convenient operation. It provides us a more intelligent detection way to measure the atmospheric quality elsewhere. The test results show that this system working stability, high accuracy. It can be used in the energy conservation and emissions reduction, environmental protection and other aspects of the application in the future.

\section{Acknowledgement}

This work was partly supported by the Shaanxi Provincial Department of education scientific research project (No. 15JK1676) of China, the project (2015EA03) supported by the key project of social science planning of Xi'an City and the Youth Founded Project of Xi'an University of Posts and Telecommunications (101-0488).

\section{References}

[1] D.V. Vladutescu, Y.H. Wu and Gross, M. Barry. Instrumentation and Measurement, No.6 (2012), p.1733-1746.

[2] S.C. Folea, G. Mois, Sensors Journal, No.9 (2015), p.742-749.

[3] H. Yang,Y. Qin, and G.F. Feng. Sensors Journal, Vol. 13(2013), No. 2, p.556-562. 
[4] R. Agarwal, S. Kumar and R.M. Hegde. Sensors Journal, Vol. 15(2015), No. 3, p.1920-1930.

[5] A. Kumar, G.P. Hancke. Sensors Journal, Vol. 14(2014), No. 8, p.2557-2566.

[6] J. Schmalzel, F. Figueroa and R. Polikar. Instrumentation and Measurement, Vol. 54(2005), No. 8, p.1612-1616.

[7] W.T. Sung, C.C. Hsu. Communications, IET, Vol. 7(2013), No. 16, p.1789-1801.

[8] C.O. Adika, L.F. Wang. Smart Grid, Vol. 5(2014), No. 2, p.673-682.

[9] C.H. Kim, M.J. Beliatis and K.K. Gandhi. Photovoltaics, Vol. 5 (2015), No. 7, p.1100-1105.

[10] V. Jelicic, M. Magno and D. Brunelli. Sensors Journal, Vol. 13 (2013), No. 1, p.328-338,

[11]D.S. Ghataoura, J.E. Mitchell and G.E. Matich, Communications Magazine, Vol. 49(2011), No. 10, pp.90-97.

[12] S. Yang, X.Y. Yang and J.A. McCann. Selected Areas in Communications, Vol. 31(2013), No. 12 , p.750-761.

[13] S. Ghosh, H.T. Wang and W.D. Leon-Salas Power Electronics, Vol. 29(2014), No. 9, p.4658-4671. 